\title{
Bacterial Food Borne Diseases
}

\section{Fahim Shaltout*}

Professor of Food Hygiene, Faculty of Veterinary Medicine, Benha University, Egypt

*Corresponding Author: Fahim Shaltout, Professor of Food Hygiene, Faculty of Veterinary Medicine, Benha University, Egypt.

Received: September 23, 2019; Published: October 10, 2019

DOI: 10.31080/ASNH.2019.03.0493

Social well being and nutrition

Meat is an excellent sources of high quality animal protein, vitamins and certain minerals, especially iron.

Meat products such as sausage, luncheon and minced meat are more popular because they are considered as a quick easily prepared meat meals and solve the problem of fresh meat shortage which is not within the reach of many peoples.

Bacteria may contaminate meat products during a long chain of processing from the time of preparation, handling, processing, distribution and storage as well as marketing. Such contamination may render the products of inferior quality or even unfit for human consumption and at times may constitute a public health hazard. Possibility of contamination of meat products with food poisoning bacteria especially Salmonella organisms has been extensively reported.

Food borne diseases are considered as diseases either infectious or toxic made by causative agents in ingested food. The reports in 2005 recorded 1.8 million people died from diseases causing diarrhea and high proportion of which was attributed to contamination of food and drinking water.

Salmonella food poisoning is one of the most common and widely distributed diseases in the world, estimated to cause 1.3 billion cases of gastroenteritis and 3 million deaths worldwide.

For the last two decades, Salmonella was considered the most common food borne pathogen in the world because Salmonellosis incidence has been constantly increasing.

Recently, some food borne diseases are considered as emerging diseases. Various food borne pathogens have been identified for food borne illness. Campylobacter, Escherichia coli 0157:H7, Listeria monocytogenes and Salmonella are found to be responsible for most of food origin outbreaks.

Traditional and conventional methods for identification of microbial pathogens depend on specific bacteriological and biochemical identification, which are time consuming, laborious and less accurate and slow because Salmonella needs 5 - 7 days to confirm its presence in meat products.
Monoclonal antibodies have been used in the diagnosis of food borne pathogens as Salmonella enterica, Salmonella typhi.

Most of immunological tests used for detection of foodborne pathogens are agglutination test western blot test, enzyme immunoassay, enzyme linked immunosorbent assay. The sensitivity and specificity of the polymerase chain reaction is very high as it is able to detect even single bacterium that's because it detects a single copy of targeted sequence of DNA, amplification of the target rather than the signal makes it promising, and lowers the liability of falsepositive results as well as lower time required for performing the technique.

Several polymerase chain reaction assays have been developed by targeting various Salmonella genes, such as invA, The invA gene in particular is widely used as a target in PCR assays for Salmonella detection.

\section{Volume 3 Issue 11 November 2019 (C) All rights are reserved by Fahim Shaltout.}

